\title{
Family carers' distress and abusive behaviour: longitudinal study
}

Claudia Cooper, Martin Blanchard, Amber Selwood, Zuzana Walker and Gill Livingston

\section{Background}

A third of family carers of people with dementia report abusive behaviour towards the person for whom they are caring. This is the first longitudinal study to investigate such behaviour

\section{Aims \\ To test our hypotheses that carers' reports of abusive behaviour would increase over time, and that change in abuse scores would be predicted by change in anxiety and depression scores. \\ Method \\ In total, 131 (71.6\%) of the family/friend dementia carers consecutively recruited from new referrals to Essex and London community mental health teams who were interviewed at baseline, completed the revised Modified Conflict Tactics scale to measure abuse 1 year later.}

\section{Results}

Sixty-three (48.1\%) of the carers reported any abusive behaviour at baseline compared with $81(61.8 \%)$ a year later $\left(\chi^{2}=6.9, P=0.009\right)$. An increase in abuse scores was predicted by an increase in anxiety and depressive symptoms (respectively $\beta=0.32, t=3.9, P<0.001$ and $\beta=0.24, t=2.9, P=0.005)$, and by less domiciliary care at baseline $(\beta=-0.18, t=-2.2, P=0.031)$.

\section{Conclusions}

Most abusive behaviour reported by carers at baseline persisted or worsened in the following year, despite contact with specialist services. We suggest that trials of psychological interventions shown to reduce anxiety and depression in the carers of people with dementia are needed to determine whether they also reduce elder abuse, and can be delivered cost-effectively within the National Health service (NHS).

\section{Declaration of interest}

None.
Abusive behaviour is commonly reported by carers of people with dementia. We previously reported in the CARD (Caring for Relatives with Memory Disorders) study that a third of family carers of people referred to psychiatric secondary care described significant levels of abuse, and half some abusive behaviour. ${ }^{1}$ These rates were comparable with other studies of abuse reported by family carers of people with dementia. ${ }^{2}$ Elder abuse is defined as a single or repeated act or lack of appropriate action occurring within any relationship where there is an expectation of trust, which causes harm or distress to an older person. ${ }^{3}$

The National Dementia Strategy ${ }^{4}$ outlines the government's 10-year plan to improve the quality of care for people with dementia and their carers. Good-quality care should include prevention of abuse, but currently there are no evidence-based interventions, possibly because a dearth of research exploring the causes of elder abuse prevents their development. Higher levels of abuse were associated with higher carer anxiety and depression in our study of family carers for people with dementia ${ }^{5}$ and a US study in a convenience sample of frail older adults and their carers. ${ }^{6,7}$

No previous longitudinal study has investigated the prevalence of elder abuse, so we do not know whether these abusive acts tend to persist or worsen over time, or whether after diagnosis and interventions they tend to improve. We know that levels of anxiety and depression in carers of people with dementia tend to increase over time. ${ }^{8}$ We hypothesise that anxiety and depression lead to carers acting more abusively, because they view the current or future situation more negatively or are less able to cope with the demands of caring or to ask for help. If this is so, we would expect carers in general also to report more abusive behaviour over time and for this to be predicted by increasing anxiety and depression.

In the current study, we measured the prevalence of abusive behaviour 1 year after our initial survey and which took place among carers of people with dementia consecutively referred to secondary services. We tested our hypotheses that carers' reports of abusive behaviour would increase over time, and that change in abuse scores would be predicted by change in anxiety and depression scores, after considering potential confounders.

\section{Method}

\section{Setting and participants}

At baseline, we recruited consecutively referred family carers of people with a clinical diagnosis of dementia; living at home and referred to community mental health teams covering London and Essex. They included inner city, suburban and rural areas. We defined a family carer as providing care for $\geqslant 4 \mathrm{~h}$ a week. Recruitment took place between January 2007 and May 2008. Interviews were at a time and place convenient to the carer, usually their home.

\section{Data collection}

The study received research ethics committee approval. Participants gave written informed consent. The information sheet specified that 'we respect confidentiality but cannot keep it a secret if anyone is being seriously harmed'. Care recipients were asked for consent to access their medical notes, but not interviewed. Where the person with dementia lacked capacity to consent (as judged from their psychiatrist and carer's reports), we asked carers whether they thought that the individual would have agreed when they had capacity. Three experienced psychiatrists carried out baseline interviews between January 2007 and April 2008.

The first author (C.C.) contacted the participants 1 year after they had participated, unless the care recipient was known to have 
died. Participants were asked whether they were still in regular contact with the person they were caring for a year ago, and if so they were asked to complete a follow-up interview. Four participants asked to complete the questionnaire as an interview, two face to face and two by telephone; the remainder replied by post. The questionnaire was re-sent once if no response had been received within a month of the first mailing.

\section{Baseline measures}

We collected sociodemographic data on: carer and care recipient age, gender, ethnicity, marital status, qualifications and alcohol use; relationship to the person being cared for (spouse, child, other); hours caring per week over preceding few weeks (recorded as 168 if the co-resident carer reported caring all the time and could not leave); care recipient's accommodation; and whether the carer worked.

\section{Modified Conflict Tactics Scale}

Our main outcome measure was the validated Modified Conflict Tactics Scale (MCTS), ${ }^{9,10}$ asking how often in the past 3 months they had acted in each of five psychologically and five physically abusive ways towards the person with dementia on a Likert scale from 0 to 4 (never, almost never, sometimes, most of the time, all the time). A score of $\geqslant 2$ (sometimes) on any question denotes significant abuse. We used the total abuse score in our analyses. The psychological abuse items asked were whether they had: screamed or shouted at the care recipients, used a harsh tone of voice, insulted, swore at them or called them names; threatened to send them to a care home, to stop taking care of or abandoning them; or used physical force on them. The physical abuse items were: whether they had been afraid they might hit or hurt them, whether they had withheld food, hit or slapped, shaken or handled them roughly in other ways. We have previously reported that the MCTS was acceptable and had good convergent and discriminant validity and internal consistency for measuring abusive behaviour by carers of people with dementia, and was much better than observer-reported measures at detecting abuse. ${ }^{9}$ To minimise bias, the MCTS was completed by the carer, unless they were unable to do so, in which case the researcher read out the questions and the possible responses and recorded their answers. We have directly compared objective measures of detecting elder abuse, which ask assessors to look for signs of abuse, with carer self-report measures, and found the latter to be more sensitive. ${ }^{9}$ The MCTS is not designed to detect abusive behaviour that the carer wishes to hide, although the relatively high proportion of carers reporting abuse suggests it is good at enabling people to divulge abuse.

\section{Other measures}

Carers also completed the following validated measures.

Hospital Anxiety and Depression Scale. The Hospital Anxiety and Depression Scale (HADS) $)^{11,12}$ has previously been used for carers of people with dementia. It does not include somatic items (which can be misleading in older people) and has been validated throughout the age range and in all settings to identify clinically significant anxiety and depression. ${ }^{12}$ It assesses how the person has been feeling within the past week. It consists of two seven-item subscales, each score $0-3$, which generate scores for generalised anxiety and depression (0-21).

The Brief Coping Orientations to Problems Experienced (COPE) scale. This is a self-report questionnaire with 14 subscales describing different coping strategies (with two items per scale). ${ }^{13}$
Carers were asked to score each strategy from 1 (not doing it at all) to 4 (doing it a lot). We used three subscales of the COPE for which adequate psychometric properties in dementia carers are reported: ${ }^{14}$ problem-focused (active coping, instrumental support and planning), emotion-focused (acceptance, emotional support, humour, positive reframing and religion) and dysfunctional coping (behavioural disengagement, denial, selfdistraction, self-blame, substance use and venting).

Zarit Burden scale. The Zarit Burden scale ${ }^{15}$ for carer burden is a 22-item self-report questionnaire and is the most consistently used measure of carer burden. It has a high internal consistency and construct validity. ${ }^{16}$ 'Carer burden' may be divided into the physical, psychological, social and financial demands of caring for someone, or into 'subjective' and 'objective' burden, with subjective burden referring to the emotional consequences of caregiving.

Health Status Questionnaire (HSQ-12). This is a quality-of-life instrument and it is valid and reliable throughout the adult age range and three of its domains measure physical health: health perception, role physical and physical functioning. ${ }^{17,18}$ For health perception, carers were asked to rate their health on a Likert scale from 1 (excellent) to 5 (poor). For physical functioning, they were asked about how their health limited activities such as climbing stairs, a higher score indicating fewer physical limitations. For impact on activities, people were asked how their physical health problems limited them in their daily activities, and asked to score this on a five-point Likert scale, from 'limited a little' to 'a great deal'.

Carers were asked about their life events in the past 6 months using the List of Threatening Experiences. ${ }^{19}$ Finally, they were asked about how rewarding their relationship with the care recipients was before the onset of memory problems, using a four-item measure of relationship rewards for which reliability in dementia carers is reported. ${ }^{20}$

Additional measures. Carers were also asked to complete the Alzheimer's Disease Co-operative Study Inventory - activities of daily living (ADCS-ADL) scale, ${ }^{21}$ and the Neuropsychiatric Inventory (NPI) to measure neuropsychiatric symptoms. ${ }^{22}$ Carers completed the MCTS (see above), modified to include abusive behaviour by the care recipients towards them. We asked when the care recipients first had memory problems and the number of alcohol units they drank per week. We recorded the care recipients' service receipt using the Client Service Receipt Inventory, ${ }^{23}$ and calculated hours of community healthcare; in-home and out-of-home social care; and days in hospital for the preceding 3 months. We asked carers about the current physical health problems of the person receiving care. We obtained the most recent Mini-Mental State Examination (MMSE) ${ }^{24}$ score and medications from the care recipients' medical notes.

\section{Follow-up interview}

Carers were asked whether the person they cared for was now living in 24-hour care, and to complete again the measures of abusive behaviour towards the care recipient, anxiety and depression.

\section{Data analysis}

We compared sociodemographic and morbidity characteristics of those interviewed at baseline who took part in the follow-up interview with those who did not. We compared anxiety, depression and abuse scores between baseline and 1-year follow-up, and the 
proportion of carers reporting any abuse and scoring as abuse cases using appropriate univariate tests, and calculated the change in each of these scores over this time.

We explored the univariate relationship between change in abuse score and sociodemographic, carer and care recipients' characteristics at baseline, and anxiety and depression change scores. We transformed the two variables entered into the multivariate analyses that had a skew statistic $>1$ or $<-1$ before the regression analysis (to $\log$ of variable +1 ). These were: length of memory problems and in-home social care (post transformation skew statistics were -0.25 , s.d. $=0.21$ and 0.91 , s.d. $=0.21$ respectively). We conducted a forwards linear regression with change in abuse score as the dependent variable. We entered all the characteristics studied that approached significance $(P<0.1)$ in their relationship with change in abuse score.

\section{Results}

In total, 220/319 (69\%) of eligible carers participated in baseline interviews, 99 refused or were uncontactable. There were no differences between participants and non-participants in care recipient or carer gender $\left(\chi^{2}=1.3, P=0.26: \chi^{2}=0.0, P=1.00\right)$, whether they lived together $\left(\chi^{2}=2.2, P=0.14\right)$ or the relationship (partner, child or other) between them $\left(\chi^{2}=2.7, P=0.26\right)$.

At 1 year, 28 dyads did not complete the questionnaire because the person with dementia had died, 1 because the carer had died, and 8 because they no longer saw the person with dementia frequently. Of the 183 remaining carers who were eligible for follow-up, $131(71.6 \%)$ completed the follow-up questionnaire. These 131 carers did not differ from the 89 non-participants in terms of care recipients' gender $\left(\chi^{2}=0.31, P=0.58\right)$, carer gender $\left(\chi^{2}=0.05, P=0.83\right)$, relationship between them (spouse, child or other) $\left(\chi^{2}=2.8, P=0.25\right)$ and whether they lived together $\left(\chi^{2}=0.04, P=0.95\right)$. There were also no differences in baseline scores on the NPI (Mann-Whitney $U$-test $=5147.0, P=0.14$ ), MMSE $(U=4658.5, P=0.10)$, ADL scale $(t=0.50, P=0.62)$, carer abusive behaviour scale $(U=5164.0, P=0.13)$, burden $(t=0.15$, $P=0.88)$, anxiety $(t=0.51, P=0.61)$ or depression scores $(t=0.83$, $P=0.41)$. There were also no differences between those who took part in the follow-up and those who did not in terms of their use of problem-focused $(t=-0.08, P=0.94)$, emotion-focused $(t=0.51, P=0.61)$ or dysfunctional coping strategies $(U=5784.5$, $P=0.92)$. In the rest of the analyses, we included only the 131 carers who completed the follow-up measures.

\section{Univariate analyses}

Mean scores were higher at follow-up on measures of carer abusive behaviour: mean 1.8 (s.d.=2.1) v. 1.3 (s.d. $=1.8$ ) at baseline (Wilcoxon signed-rank test statistic $z=2.9, P=0.003$ ); depression (6.0 (s.d.=4.3) v. $4.9($ s.d.=4.4) $z=3.4, P=0.001)$ and anxiety $(8.2($ s.d. $=4.4)$ v. $6.3($ s.d. $=4.3))$; paired $t$-test $=4.9$, $P<0.001)$. In total, $41(31.5 \%)$ carers scored as abuse cases at baseline compared with $45(34.4 \%) 1$ year later (McNemar's test $\left.\chi^{2}=0.11, P=0.74\right)$, and $63(48.1 \%)$ of carers reported any abusive behaviour at baseline compared with $81(61.8 \%)$ a year later $\left(\chi^{2}=6.9, P=0.009\right)$.

Of the baseline sociodemographic characteristics studied, only the recipients of care without qualifications were associated with change in abuse score $(0.68$ (s.d. $=1.9)$ increase v. $0.13($ s.d. $=1.8)$ decrease in those caring for people with qualifications (Table 1)).

A greater increase in abuse score was also associated with a shorter duration of memory problems, receiving fewer hours of in-home social care at baseline and increases in depression and anxiety scores (Table 2).
Table 1 Categorical sociodemographic characteristics and their relationship with change in abuse score over 1 year

\begin{tabular}{lcccc} 
& $n(\%)$ & Test & \\
Category & $(n=131)$ & statistic & $P$ \\
Care recipients & & & \\
Female & $97(74.0)$ & $t=0.83$ & 0.41 \\
Ethnicity: White, UK & $105(80.2)$ & $t=1.1$ & 0.26 \\
Living with carer & $71(54.2)$ & $t=0.81$ & 0.42 \\
Accommodation at baseline & & $F=0.94$ & 0.51 \\
$\quad$ Home owner & $76(58.0)$ & & \\
$\quad$ Renting & $43(32.8)$ & & \\
$\quad$ Sheltered & $12(9.2)$ & & \\
Entered care home during follow-up year & $18(13.7)$ & $t=1.1$ & 0.29 \\
Married/cohabiting & $56(42.7)$ & $t=0.02$ & 0.98 \\
No qualifications & $101(77.1)$ & $\boldsymbol{t}=2.1$ & $\mathbf{0 . 0 3 8}$ \\
\hline Carer & & & \\
Female & $87(66.4)$ & $t=1.3$ & 0.20 \\
Ethnicity: White, UK & $109(83.2)$ & $t=0.01$ & 0.99 \\
Relationship to care recipient & & $F=0.61$ & 0.83 \\
$\quad$ Spouse & $48(36.6)$ & & \\
$\quad$ Child & $69(52.7)$ & & \\
$\quad$ Other relative or friend & $14(10.7)$ & & \\
Married/cohabiting & $97(74.0)$ & $t=1.1$ & 0.30 \\
Qualifications & & $F=0.94$ & 0.51 \\
$\quad$ None & $45(34.4)$ & & \\
O-level & $34(26.0)$ & & \\
A-level & $25(19.1)$ & & \\
$\quad$ Degree + & $27(20.6)$ & & \\
Working & & $F=1.1$ & 0.36 \\
$\quad$ No & $83(63.4)$ & & \\
Part time & $19(14.5)$ & & \\
Full time & $29(22.1)$ & & \\
Results in bold are statistically significant. & & & \\
\hline & & & \\
\hline
\end{tabular}

\section{Multivariate analysis}

We entered change in anxiety score and all other characteristics that approached significance $(P<0.1)$ in their relationship with change in abuse score, in a forwards linear regression, with change in abuse score as a dependent variable. Table 3 shows the correlation matrix for variables included. Changes in anxiety and depression scores were highly correlated, so were not included in the same equation.

Only an increase in anxiety score (standardised $\beta=0.32$, $t=3.9, \quad P<0.001)$ and less in-home social care at baseline $(\beta=-0.18, t=-2.2, P=0.031)$ were associated with an increase in abuse score (adjusted $R^{2}=0.13$ ). We repeated the above analysis including change in depression rather than anxiety score. The significant predictors were an increase in depression score ( $\beta=0.24, t=2.9, P=0.005)$, less in-home social care at baseline $(\beta=-0.19, t=-2.3, P=0.025)$ and the care recipients having more qualifications at baseline $(\beta=-0.18, t=-2.2, P=0.028)$ (adjusted $\mathrm{R}^{2}=0.12$ ).

\section{Discussion}

Around half of carers reported any abusive behaviour at baseline, and this had increased to nearly two-thirds a year later. Most abusive behaviour reported by carers at baseline persisted or worsened in the following year, despite their contact with specialist services. Our findings suggest that current dementia services are not adequately addressing carer abusive behaviour among people with dementia living at home, and that targeted interventions are needed. 


\begin{tabular}{|c|c|c|c|c|c|}
\hline Characteristics & Mean (s.d.) & Median (range) & Skewness statistic & Correlation $^{\mathrm{a}}$ & $P$ \\
\hline \multicolumn{6}{|l|}{ Sociodemographic factors } \\
\hline Care recipient age & $81.2(7.6)$ & 82 (58 to 95$)$ & -0.79 & 0.09 & 0.53 \\
\hline Carer age & $62.8(12.6)$ & 61 (32 to 92) & 0.17 & 0.08 & 0.37 \\
\hline Hours of care provided a week & $98.4(76.1)$ & 168 (4 to 168$)$ & -0.21 & 0.05 & 0.55 \\
\hline \multicolumn{6}{|l|}{ Care recipient morbidity } \\
\hline Mini-Mental State Examination $(n=127)$ & $19.0(6.5)$ & 20.0 (0 to 29$)$ & -1.310 & -0.04 & 0.68 \\
\hline Neuropsychiatric Inventory & $16.7(14.5)$ & $14(0$ to 69$)$ & 1.19 & -0.08 & 0.39 \\
\hline ADCS-ADL & $19.6(12.8)$ & 17 (0 to 54$)$ & 0.59 & -0.10 & 0.28 \\
\hline Abusive behaviour to carer score & $2.0(3.1)$ & 0 (0 to 14$)$ & 2.01 & -0.12 & 0.19 \\
\hline Number of physical illnesses & $2.5(1.5)$ & $2(0$ to 7$)$ & 0.51 & -0.02 & 0.80 \\
\hline Number of medications & $4.4(3.1)$ & $4(0$ to 16$)$ & 0.90 & 0.11 & 0.23 \\
\hline Months with memory problems & $36.1(42.0)$ & 24 (0 to 300$)$ & 3.40 & -0.19 & 0.032 \\
\hline Units of alcohol per week & $3.8(10.2)$ & $0(0$ to 84$)$ & 4.94 & 0.01 & 0.92 \\
\hline \multicolumn{6}{|l|}{ Hours of formal care in past 3 months } \\
\hline In-home social care & $40.0(97.5)$ & $0(0$ to 624$)$ & 3.59 & -0.17 & 0.055 \\
\hline Social care outside home & $17.6(80.8)$ & $0(0$ to 845$)$ & 8.67 & -0.06 & 0.49 \\
\hline Healthcare & $4.4(11.9)$ & $1.8(0.2$ to 107$)$ & 6.7 & -0.06 & 0.50 \\
\hline \multicolumn{6}{|l|}{ Carer factors (baseline) } \\
\hline Health perception & $2.7(1.2)$ & $3(1$ to 5$)$ & 0.23 & 0.09 & 0.33 \\
\hline Role physical & $1.9(1.2)$ & 1 (1 to 5$)$ & 1.09 & 0.07 & 0.42 \\
\hline Physical functioning & $2.6(0.64)$ & 3 (1 to 3$)$ & -1.30 & -0.10 & 0.27 \\
\hline Burden score & $29.6(16.3)$ & 29 (0 to 84$)$ & 0.56 & -0.09 & 0.29 \\
\hline Life events & $0.53(0.78)$ & 0 (0 to 3$)$ & 1.40 & 0.06 & 0.48 \\
\hline COPE dysfunctional & $17.6(5.2)$ & $16(12$ to 41$)$ & 1.42 & -0.09 & 0.36 \\
\hline COPE emotion-focused & $21.0(5.5)$ & $21(10$ to 38$)$ & 0.31 & -0.10 & 0.26 \\
\hline COPE problem-focused & $14.7(4.9)$ & 15 (6 to 24$)$ & 0.11 & -0.12 & 0.16 \\
\hline Units of alcohol & $3.8(10.2)$ & 0 (0 to 84) & 4.94 & 0.06 & 0.50 \\
\hline Previous relationship rewards $(n=128)$ & $9.5(2.9)$ & 11 (1 to 12$)$ & -0.95 & 0.07 & 0.41 \\
\hline \multicolumn{6}{|l|}{ Carer factors (change from baseline to 1 year) } \\
\hline Anxiety & $1.87(4.4)$ & $2(-9$ to 16$)$ & 0.26 & 0.34 & $<0.001$ \\
\hline Depression & $1.15(4.3)$ & $1(-19$ to 10$)$ & -0.76 & 0.27 & 0.002 \\
\hline
\end{tabular}

\begin{tabular}{|c|c|c|c|c|c|}
\hline & \multicolumn{5}{|c|}{ Correlation coefficient $(P)$} \\
\hline & $\begin{array}{l}\text { Change in } \\
\text { abuse score }\end{array}$ & $\begin{array}{l}\text { Change in } \\
\text { anxiety score }\end{array}$ & $\begin{array}{c}\text { Change in } \\
\text { depression score }\end{array}$ & $\begin{array}{l}\text { Time with memory } \\
\text { problems }\end{array}$ & $\begin{array}{c}\text { Care recipients } \\
\text { having qualifications }\end{array}$ \\
\hline Change in anxiety score & $0.34(<0.001)$ & & & & \\
\hline Change in depression score & $0.27(0.002)$ & $0.60(<0.001)$ & & & \\
\hline Time with memory problems ${ }^{a}$ & $-0.19(0.032)$ & $0.07(0.46)$ & $0.08(0.35)$ & & \\
\hline Care recipients having qualifications ${ }^{a}$ & $-0.12(0.17)$ & $-0.14(0.11)$ & $0.04(0.64)$ & $0.03(0.74)$ & \\
\hline In-home social care ${ }^{a}$ & $-0.17(0.055)$ & $-0.07(0.43)$ & $-0.10(0.25)$ & $0.18(0.04)$ & $-0.03(0.75)$ \\
\hline
\end{tabular}

The increase in carer abusive behaviour over a year was strongly predicted by an increase in depression and anxiety symptoms. Perhaps carers who felt they had lost the person they knew to dementia were more anxious and depressed, and also more likely to abuse. Alternatively, carer anxiety and depression may have been associated with a lack of confidence in their ability to care, and thus greater use of abusive rather than more helpful strategies for managing difficulties. Anxiety and depression may also have lowered the ability of carers to manage difficulties that arose, so they were more likely to feel overwhelmed and react abusively.

This is the first longitudinal study to explore the relationship between carer mental health and abusive behaviour. We only measured abuse, anxiety and depression at baseline and 1 year. We cannot determine whether onset of abusive behaviour occurred before or after anxiety or depressive symptoms from this study design. Therefore, although we explored change over time in these scores, and demonstrated our hypothesis that change in abusive behaviour was predicted by change in anxiety and depression, it is also possible that abusive behaviour led to anxiety and depression. It is plausible that guilt should arise from abusive acts, or that worry regarding the consequences of abuse could lead to carer anxiety and depression.

The National Institute for Health and Clinical Excellence and the Social Care Institute for Excellence dementia care clinical guidelines recommend that 'Carers of people with dementia who experience psychological distress and negative psychological impact should be offered psychological therapy, including cognitive behavioural therapy, conducted by a specialist 
practitioner. ${ }^{25}$ In reality, resources are not available, although there is a recent precedent for broadening availability of psychological interventions. In 2008, the UK Department of Health unveiled plans to train an extra 3600 psychological therapists to treat depression and anxiety in primary care, with training and supervision from clinical psychologists. ${ }^{26}$ Such a model could also be employed to deliver psychological therapies adapted for carers of people with dementia. These may include interventions to encourage use of helpful coping strategies, and discourage unhelpful strategies, when managing the problems in caring for someone with dementia. ${ }^{27}$ As levels of anxiety, depression and abusive behaviour in dementia carers increased over time, with nearly two-thirds reporting any abusive behaviour at follow-up, such interventions could usefully be offered to all family carers of people newly diagnosed with dementia.

Such a programme would be expensive and would need careful piloting to include an evaluation of the benefits and cost-effectiveness of implementation. There is good evidence from the USA that manualised psychological interventions can improve the mental health of dementia carers. ${ }^{28}$ We think these need to be evaluated urgently to determine whether they can be effectively delivered across the National Health Service (NHS). Our findings suggest that tackling carer anxiety and depression may also reduce abusive behaviour by carers, but further work is needed to test this hypothesis. There is also evidence from the USA that providing specialist, individually tailored psychological support to people with dementia and their family carers can reduce rates of institutionalisation. ${ }^{29}$ Elder abuse has been associated with a greater likelihood of an older person moving to a care home, ${ }^{30}$ although we did not find this in our sample, possibly because of decreased power to detect this. Nationally, a reduction in carehome placements would have huge benefits for society because most older people want to continue living at home and report higher quality of life when they do so, compared with those placed in care homes. There are also economic benefits; the National Audit Office recently emphasised the need to 'spend to save' on dementia care. $^{4}$

Receiving fewer hours of in-home social care at baseline was also associated with an increase in abuse score. This included care worker, social worker and in-home respite time. This suggests that in-home services may be particularly important in reducing abuse, although it is also possible that this relationship is confounded, as people with dementia who have more behavioural and psychological symptoms of dementia are less likely to accept home care and, as they are more difficult to care for, their carers may be more stressed and therefore act more abusively.

\section{Limitations}

We measured service use at baseline but not at follow-up. We therefore do not know how this may have changed over the year, and it is possible that loss of services may have lead to anxiety, depression and abusive behaviour in some carers. This does not alter our finding that change in affective symptoms predicted change in abusive behaviour. Just under a third of carers approached refused to participate in the study. This compares positively with response rates in recent large general population surveys; $57 \%$ of people approached for the 2007 Adult Psychiatric Morbidity Survey agreed to take part, ${ }^{31}$ suggesting that carers were not dissuaded from taking part because of the nature of the interview. Nonetheless, this is a limitation as we do not know whether those who refused to take part would have been more or less likely to report abuse. As the information sheet explained that confidentiality may be breached if someone was being harmed, it is perhaps more likely that those who were abusing would refuse follow-up. Similarly, the carers who completed the baseline interview but did not return the follow-up questionnaire may have been more stressed, or more likely to be acting abusively than those who did return it. Most of the carers completed the first assessment at interview and the second in a postal survey. They may have been more willing to disclose abuse by post. There may be other factors we have not considered that confound or explain the relationship between abuse and psychological morbidity.

Claudia Cooper, PhD MRCPsych, Martin Blanchard, MD MRCPsych, Ambe Selwood, MSC MRCPsych, Zuzana Walker, MD FRCPsych, Gill Livingston, MD FRCPsych, Department of Mental Health Sciences, University College London, UK.

Correspondence: Claudia Cooper, PhD, MRCPsych, Department of Mental Health Sciences, UCL, 67-73 Riding House Street, 2nd Floor, Charles Bell House, London W1W 7EJ, UK. Email: c.cooper@ucl.ac.uk

First received 10 Aug 2009, final revision 25 Jan 2010, accepted 9 Feb 2010

\section{Funding}

The study was funded by a Medical Research Council research training fellowship awarded to C.C.

\section{Acknowledgements}

We would like to thank the carers and patients who took part in the study. We are grateful to Camden and Islington NHS Foundation Trust, North Essex Partnership Foundation NHS Trust and North East London Mental Health Trust; in particular to Drs Allen, Joels, Kirchner and Stevens. We would like to thank Dr Colm Owens for conducting some of the interviews and the Alzheimer's Society (Islington Branch) and Shirley Nurock for her assistance in developing the study.

\section{References}

1 Cooper C, Selwood A, Blanchard M, Walker Z, Blizard R, Livingston G. Abuse of people with dementia by family carers: representative cross sectional survey. BMJ 2009; 338: 155.

2 Cooper C, Selwood A, Livingston G. The prevalence of elder abuse and neglect: a systematic review. Age Ageing 2008; 37: 151-60.

3 House of Commons Health Select Committee. Elder Abuse. No. 2. TSO (The Stationery Office), 2004.

4 Department of Health. The National Dementia Strategy. TSO (The Stationery Office), 2008.

5 Cooper C, Selwood A, Blanchard M, Walker Z, Blizard R, Livingston G. The determinants of family carer's abusive behaviour to people with dementia: results of the CARD study. J Affect Disord 2010; 121: 136-42.

6 Beach SR, Schulz R, Williamson GM, Miller LS, Weiner MF, Lance CE. Risk factors for potentially harmful informal caregiver behavior. J Am Geriatr Soc 2005; 53: 255-61.

7 Shaffer DR, Dooley WK, Williamson GM. Endorsement of proactively aggressive caregiving strategies moderates the relation between caregiver mental health and potentially harmful caregiving behavior. Psychol Aging 2007; 22: 494-504.

8 Cooper C, Katona C, Orrell M, Livingston G. Coping strategies, anxiety and depression in caregivers of people with Alzheimer's disease. Int J Geriatr Psychiatry 2008; 23: 929-36.

9 Cooper C, Manela M, Katona C, Livingston G. Screening for elder abuse in dementia in the LASER-AD study: prevalence, correlates and validation of instruments. Int J Geriatr Psychiatry 2008; 23: 283-8.

10 Beach SR, Schulz R, Williamson GM, Miller LS, Weiner MF, Lance CE. Risk factors for potentially harmful informal caregiver behavior. J Am Geriatr SoC 2005; 53: 255-61.

11 Zigmond AS, Snaith RP. The Hospital Anxiety and Depression Scale. Acta Psychiatr Scand 1983; 67: 361-70.

12 Bjelland I, Dahl AA, Haug TT, Neckelmann D. The validity of the Hospital Anxiety and Depression Scale: an updated literature review. J Psychosom Res 2002; 52: 69-77.

13 Carver CS. You want to measure coping but your protocol's too long: consider the brief COPE. Int J Behav Med 1997; 4: 92-100. 
14 Cooper C, Katona C, Livingston G. Validity and reliability of the brief COPE in carers of people with dementia - the LASER-AD study. J Nerv Ment Dis 2008; 196: 838-43.

15 Zarit SH, Reever KE, Bachpeterson J. Relatives of the impaired elderly correlates of feelings of burden. Gerontologist 1980; 20: 649-55.

16 Hebert R, Levesque L, Vezina J, Lavoie JP, Ducharme F, Gendron C, et al. Efficacy of a psychoeducative group program for caregivers of demented persons living at home: a randomised controlled trial. J Gerontol B Psychol Sci SOC Sci 2003; 58: S58-67.

17 Radosevich D, Pruitt M. Twelve-Item Health Status Questionnaire: HSQ-12 Version 2.0. Health Outcomes Institute, 1995.

18 Pettit T, Livingston G, Manela M, Kitchen G, Katona C, Bowling A. Validation and normative data of health status measures in older people: the Islington study. Int J Geriatr Psychiatry 2001; 16: 1061-70.

19 Brugha $\mathrm{T}$, Bebbington $\mathrm{P}$, Tennant $\mathrm{C}$, Hurry J. The List of Threatening Experiences - a subset of 12 life event categories with considerable longterm contextual threat. Psychol Med 1985; 15: 189-94.

20 Williamson GM, Shaffer DR. Relationship quality and potentially harmful behaviors by spousal caregivers: how we were then, how we are now. Psychol Aging 2001; 16: 217-26.

21 Galasko D, Bennett D, Sano M, Ernesto C, Thomas R, Grundman M, et al. An inventory to assess activities of daily living for clinical trials in Alzheimer's disease. The Alzheimer's Disease Cooperative Study. Alzheimer Dis Assoc Disord 1997; 11 (suppl 2): S33-9.

22 Cummings JL. The Neuropsychiatric Inventory: assessing psychopathology in dementia patients. J Gerontol B Psychol Sci Soc Sci 1997; 48: 10-16S.

23 Knapp M. The Economic Evaluation of Mental Health Care. Arena, 1995.
24 Folstein MF, Folstein SE, McHugh PR. 'Mini-mental state'. A practical method for grading the cognitive state of patients for the clinician. J Psychiatric Res 1975: 12: 189-98.

25 National Institute for Health and Clinical Excellence, Social Care Institute for Excellence. Dementia: Supporting People with Dementia and their Carers in Health and Social Care. NICE, 2006.

26 Department of Health. Improving Access to Psychological Therapies Implementation Plan: National Guidelines for Regional Delivery. Department of Health, 2008

27 Gallagher-Thompson D, Coon DW, Solano N, Ambler C, Rabinowitz Y, Thompson LW. Change in indices of distress among Latino and Anglo female caregivers of elderly relatives with dementia: site-specific results from the REACH national collaborative study. J Gerontol B Psychol Sci Soc Sci 2003; 43: 580-91.

28 Gallagher-Thompson D, Arean P, Rivera P, Thompson L. A psychoeducational intervention to reduce distress in hispanic family caregivers: results of a pilot study. J Gerontol B Psychol Sci Soc Sci 2001; 23: 17-32.

29 Mittelman MS, Roth DL, Clay OJ, Haley WE. Preserving health of Alzheimer caregivers: impact of a spouse caregiver intervention. J Gerontol B Psychol Sci Soc Sci 2007; 15: 780-9.

30 Moon A, Lawson K, Carpiac M, Spaziano E. Elder abuse and neglect among veterans in Greater Los Angeles: prevalence, types, and intervention outcomes. J Gerontol B Psychol Sci Soc Sci 2006; 46: 187-204.

31 The NHS Information Centre for Health and Social Care. Adult Psychiatric Morbidity in England, 2007 (eds S McManus, H Meltzer, T Brugha, P Bebbington, R Jenkins). Health \& Social Care Information Centre, 2009.

\section{Poems \\ by doctors}

\section{Viruses}

\section{Glenn Colquhoun}

Viruses are my sister's children

who have come to stay at my place

for the weekend.

They try to get into the cupboards.

They draw with thick crayons

on the back of my throat.

They run up and down the inside of my head with small hands covered in marmite and jam which takes forever to get off the walls.

They bang pots and pans on the floor.

They wipe their feet on the centre of my tongue.

The cry loudly in the middle of the night.

Sometimes they swing

on that dangly thing which hangs

from the back of my mouth until I cough.

I eat broken biscuits all week.

No wonder my nose runs.

Glenn Colquhoun was born in Auckland, New Zealand and is a doctor practising in Te Tai Tokeran, Northland. He was the winner of the 2003 Montana New Zealand book award for poetry. This poem is from Playing God, Steele Roberts. Another of Glenn's poems was published in the February 2010 issue of the Journal.

Poem chosen by Femi Oyebode. 